\title{
Successful implementation of best medical treatment for patients with asymptomatic carotid artery stenosis within a randomized controlled trial (SPACE-2)
}

Tilman Reiff ${ }^{*}$ (D) Hans-Henning Eckstein ${ }^{2}$, Ulrich Mansmann ${ }^{3}$, Olav Jansen ${ }^{4}$, Gustav Fraedrich ${ }^{5}$, Harald Mudra ${ }^{6}$, Werner Hacke ${ }^{1}$ and Peter Arthur Ringleb ${ }^{1}$ for the SPACE-2 study group

\begin{abstract}
Background: Asymptomatic carotid artery stenosis (ACS) can be treated with carotid endarterectomy (CEA), carotid artery stenting (CAS), or best medical treatment (BMT) only. For all treatment options, optimization of vascular risk factors such as arterial hypertension, hyperlipidemia, smoking, obesity, and insufficient physical activity is essential. Data on adherence to BMT and lifestyle modification in patients with ACS are sparse. The subject of this investigation is the implementation and quality of risk factor adjustment in the context of a randomized controlled trial.

Methods: A total of 513 patients in the prematurely terminated, randomized, controlled, multicenter SPACE-2 trial (ISRCTN 78592017) were analyzed within one year after randomization into 3 groups (CEA, CAS, and BMT only) for implementation of prespecified BMT recommendations and lifestyle modifications. Measurement time points were the screening visit and visits after one month (D30), 6 months (M6), and one year (A1). Differences between groups and follow-up visits (FUVs) relative to the screening visit were investigated.

Findings: For all FUVs, a significant increase in statin medication ( $91 \%$ at $A 1 ; p<0.0001$ ) was demonstrated to be associated with a significant decrease $(p<0.01$ ) in cholesterol levels (median $167 \mathrm{mg} / \mathrm{dl}$ at A1) and LDL cholesterol levels (median $93 \mathrm{mg} / \mathrm{dl}$ at A1). The lowest cholesterol levels were achieved by patients in the BMT group. Seventyeight percent of all patients reached predefined target cholesterol levels $(<200 \mathrm{mg} / \mathrm{dl})$, with significantly better rates in the BMT group ( $p=0.036$ at D30). Furthermore, a significant decrease in arterial blood pressure at all FUVs $(p<0.05)$ was associated with a significant increase in antihypertensive medication ( $96 \%$ at A1, $p<0.0001$ ). However, only $28 \%$ of patients achieved the predefined treatment goal of a systolic blood pressure of $\leq 130 \mathrm{mmHg}$. Forty-two of a total of 100 smokers at the screening visit quit smoking within one year, resulting in a significant increase in nonsmokers at all FUVs $(p<0.0001)$. Recommended HbA1c levels $(<7 \%)$ were achieved in $82 \%$ without significant changes after one year. Only $7 \%$ of obese (BMI > 25) patients achieved sufficient weight reduction after one year without significant changes at all FUVs (median BMI 27 at A1; $p=0.1201$ ). The BMT group showed significantly $(p=0.024)$ higher rates of adequate physical activity than the intervention groups. Furthermore, after one year, the BMT group showed a
\end{abstract}

*Correspondence: tilman.reiff@med.uni-heidelberg.de

${ }^{1}$ Department of Neurology, University Hospital of Heidelberg, Im Neuenheimer Feld 400, 69120 Heidelberg, Germany

Full list of author information is available at the end of the article original author(s) and the source, provide a link to the Creative Commons licence, and indicate if changes were made. The images or other third party material in this article are included in the article's Creative Commons licence, unless indicated otherwise in a credit line to the material. If material is not included in the article's Creative Commons licence and your intended use is not permitted by statutory regulation or exceeds the permitted use, you will need to obtain permission directly from the copyright holder. To view a copy of this licence, visit http://creativecommons.org/licenses/by/4.0/. 
comparatively significantly better implementation of risk factor modification (77\%; $p=0.027)$ according to the treating physician.

Interpretation: SPACE-2 demonstrated sustained improvement in the noninterventional management of vascular risk factors in patients treated in a clinical trial by general practitioners, internists and neurologists. The best implemented treatment targets were a reduction in cholesterol and $\mathrm{HbA1c}$ levels. In this context, a significant increase in statin use was demonstrated. Blood pressure control missed its target but was significantly reduced by intensification of antihypertensive medication. Patients on BMT only had better adjusted lipid parameters and were more physically active. However, all groups failed to achieve sufficient weight reduction. Due to insufficient patient recruitment, the results must be interpreted cautiously.

Trial registration: ISRCTN Registry, ISRCTN78592017, Registered 16 June 2007, https://www.isrctn.com/search?q= 78592017.

Keywords: Asymptomatic carotid artery stenosis, Primary prevention, Carotid endarterectomy, Carotid artery stenting, Best medical treatment, Disease-free survival, Epidemiology, Prospective study

\section{Introduction}

Patients with asymptomatic carotid artery stenosis (ACS) should be treated with best medical treatment (BMT) or in addition invasively with carotid endarterectomy (CEA) or carotid artery stenting (CAS). Regardless of the chosen treatment option, implementation of BMT and lifestyle modification are crucial for reducing the risk of cerebrovascular events. A cohort study of 2,885,257 individuals between 2003 and 2008 revealed the risk factors for the development of $\geq 50 \%$ carotid artery stenosis. Among others, uncontrolled hypertension (odds ratio (OR) 2.0; 95\% confidence interval (CI) 2.0-2.0), high cholesterol (OR 1.4; 95\% CI 1.4-1.4), diabetes (OR 1.4; 95\% CI 1.41.4), and smoking (depending on packyears, minimum OR 1.5; 95\% CI 1.5-1.6) were significant risk factors [1, 2]. These factors also play an important role in risk prediction models for the detection of ACS [3] and are not only crucial for its development but are also considered general stroke risk factors [4]. Consequently, blood pressure and diabetes control, as well as lipid-lowering medications, are considered important components of BMT [5-7]. Antiplatelet therapy has a lower level of evidence in primary prevention and may be indicated in patients with low bleeding risk and cardiovascular comorbidity [8-10]. Statins, in particular, have shown a significant impact on plaque stabilization and reduction in microembolisms with a consecutive reduced risk of vascular events [11]. Despite the clear evidence for the preventive benefits of statins, they are still underutilized $[12,13]$. A dose-dependent increase in mortality with nonadherence has been demonstrated [14]. In addition, smoking cessation, maintenance of a healthy body weight, moderate exercise, and a Mediterranean diet are essential complementary behaviors in the prevention of arterial disease not only of the brain supplying vessels but also in other arterial beds $[5-7,15,16]$. The importance of optimized $\mathrm{BMT}$ and continuously monitored and improved lifestyle modifications is considered largely underestimated by both physicians and patients [6]. To illustrate the decrease in annual ipsilateral stroke risk over the years in patients with ACS treated with BMT alone, a 2014 a metanalysis of 41 studies showed a rate of $1.0 \%$ in patients recruited between 2000 and 2009 compared to 2.3\% when recruited before $2000(p<0.001)$. Regarding statin use, the rate was $2.3 \%$ in studies with the proportion of patients receiving statins was $<25 \%$ versus a rate of $1.2 \%$ when $\geq 25 \%$ of patients received statins $(p=0.009)$ [17]. Therefore, regardless of the use of interventional or conservative therapy, adequate BMT should be implemented.

We investigated whether sufficient implementation of BMT and lifestyle modification could be achieved within one year in a randomized controlled trial. Factors with sufficient implementation could provide examples of effective management of cerebrovascular risk factors beyond clinical trials.

\section{Methods}

The international randomized, controlled, open, multicenter SPACE-2 study (ISRCTN78592017) recruited 513 patients with ACS of the common and/or internal carotid artery of $\geq 70 \%{ }^{\mathrm{ECST}}[18] \approx \geq 50 \%^{\mathrm{NASCET}}$ [19]. Patients were randomized to three treatment arms: CEA plus BMT $(\mathrm{n}=203)$, CAS plus BMT $(\mathrm{n}=197)$, and BMT alone $(n=113)$. Enrollment occurred in 36 study centers in Germany, Switzerland, and Austria. Further details and endpoints have been previously published [20]. The SPACE-2 study started in 2009. Due to insufficient recruitment rates, a change in study design was implemented in 2013 [21]. However, a persistently toolow recruitment rate despite protocol modification led to the premature termination of the study in 2014 [22]. One-year interim results of the study were published recently [23]. In this investigation, the data from the first year after randomization were analyzed regarding 
the implementation of BMT and lifestyle modification. At the screening visit (D0), after 30 days (D30), after 6 months (M6), and after one year (A1), the following examinations were recorded: blood samples with fasting glucose/HbA1c/cholesterol/LDL-cholesterol/HDLcholesterol and triglyceride levels; systolic and diastolic blood pressure, height, and weight with calculated body mass index (BMI; body mass/square of height); waistto-hip ratio (WHR; waist circumference/hip circumference). Physical activity over 30 min per week was recorded in three categories: never, 1-2 times, and 3 times or more. Smoking behavior and alcohol consumption were recorded. Medications including antiplatelet, anticoagulant, antihypertensive, lipid-lowering and antidiabetic agents and comorbidities, especially those with vascular effects such as arterial hypertension, diabetes mellitus, coronary artery disease, and hypercholesterolemia, were documented. The modified Rankin scale and National Institute of Health Stroke Scale score were assessed. Extra- and intracranial sonography of the supplying vessels of the brain was performed. Before the start of the study, BMT recommendations were established for all centers and all patient groups according to current treatment guidelines [24] (see Additional file 1: Table S1). Adjustment to more strictly defined target value ranges during the course of the study was at the discretion of the individual centers. After the screening visit, all patients received recommendations for individualized medication, optimization of lifestyle modification (nicotine withdrawal, physical activity, alcohol consumption, healthy diet), and further medical examinations (electrocardiography/echocardiography for cardiac status and ankle-brachial index for diagnosis of peripheral arterial occlusive disease). At each of the follow-up visits, the aforementioned risk factors were reviewed, and the implementation of the recommended treatment strategies was re-examined. An assessment of drug compliance was made for the lipid-lowering preparations by measuring cholesterol levels, for antihypertensives by monitoring blood pressure, and for antidiabetics by monitoring blood glucose parameters. For control of medication, the current medication list of the primary care physician and the information provided by the patient were used as a basis. Drug intolerances or side effects were evaluated, and medication adjustments were initiated if necessary. All submitted documentation of blood pressure values, laboratory parameters or hospitalizations was reviewed. All findings, adaptation of medication and goals for further reduction of vascular risk factors were explained to the patients and documented for the general practitioner. The respective study centers were responsible for providing information material, offering telephone contact to clarify problems, and recommending outpatient measures such as smoking cessation courses or other measures to support compliance.

\section{Statistical analysis}

Analysis of the rates of patients achieving the predefined BMT recommendations, blood sample parameters, blood pressure, BMI, WHR, physical activity, implementation of risk factors as assessed by the treating physician, and detailed medication was performed with the chi-square test or Fisher's exact test (if cell count $<5$ ) and further with the Mann-Whitney U or Kruskal-Wallis test, respectively. Comparisons of the values at the 30-day, one-month, and one-year visits with the baseline values at the screening visit were performed with McNemar's test and the Wilcoxon signed-rank test. A $p$ value $<0.05$ was considered statistically significant. Due to death/missing values, the total number of patients varied between the different variables; therefore, percentages were chosen as the primary measure. Since none of the analyses addressed a main target of the main study, all tests were exploratory. Analyses were performed with STATA/IC 13.0 (College Station, Texas, US).

\section{Results}

\section{Achievement of BMT targets}

See Additional file 1: Table S1 for the definition of targets for the BMT. The percentage achievement of the specified BMT targets at visits D30 to A1 is presented in Table 1. Approximately $70 \%$ of all patients did not reach the required threshold values when adjusting their blood pressure. Within one year, only a small improvement in blood pressure control was achieved, with no significant difference between the CEA, CAS and BMT groups. Cholesterol levels $<200 \mathrm{mg} / \mathrm{dl}$ were reached in $83 \%$ of the BMT group (after 30 days) and improved to $88 \%$ (after one year), with a significant difference from the CEA group (between 70\%-76\%) and the CAS group (between 77 and 79\%); (pD30 $=0.036 ; \mathrm{pM6}=0.041)$. Sufficiently lowered LDL cholesterol levels $<130 \mathrm{mg} /$ $\mathrm{dl}$ (patients without coronary heart disease (CHD)) and $<100 \mathrm{mg} / \mathrm{dl}$ (patients with CHD) were achieved by $70 \%$ (CEA at D30) to 83\% (BMT M6) of the group members without significant differences between the study groups. Sufficient control of HDL cholesterol could be achieved in $77-81 \%$ of patients without significant differences between the groups. Triglyceride target values $<150 \mathrm{mg} /$ $\mathrm{dl}$ were achieved in $58-75 \%$ of patients, with significantly better results in the BMT group after 6 months but not after one year. In all patients, HbA1c values $<7 \%$ were achieved in $81-89 \%$ of patients without significant differences between treatment groups. In the CEA group, significantly more patients had quit smoking 30 days after the screening visit (CEA: 10\%, CAS: 4\%, BMT: 
Table 1 Proportion of patients with sufficient implementation of BMT goals; visit D30 to $A 1 ; n=513$

\begin{tabular}{|c|c|c|c|c|c|}
\hline & CEA $(\%[n])$ & CAS (\% [n]) & BMT (\% [n]) & total $(\%[n])$ & $p$ \\
\hline \multicolumn{6}{|c|}{ Blood pressure $\leq 130 / 85 \mathrm{mmHg}^{1}$} \\
\hline D30 & $23.5 \%(44)$ & $26.6 \%(47)$ & $22.9 \%(24)$ & $24.5 \%(115)$ & $0.772^{\ulcorner}$ \\
\hline M6 & $25.4 \%(45)$ & $30.6 \%(53)$ & $31.1 \%(33)$ & $28.7 \%(131)$ & $0.461^{\ulcorner}$ \\
\hline A1 & $25.7 \%(44)$ & $29.8 \%(50)$ & $30.1 \%(31)$ & $28.3 \%(125)$ & $0.638^{\ulcorner}$ \\
\hline \multicolumn{6}{|c|}{ Cholesterol $<200 \mathrm{mg} / \mathrm{dl}$} \\
\hline D30 & $69.6 \%(103)$ & $79.2 \%(114)$ & $83.1 \%(74)$ & $76.4 \%(291)$ & $0.036^{\ulcorner *}$ \\
\hline M6 & $75.8 \%(116)$ & $76.5 \%(114)$ & $88.3 \%(83)$ & $79.0 \%(313)$ & $0.041^{\ulcorner *}$ \\
\hline A1 & $74.5 \%(117)$ & $77.1 \%(111)$ & $87.5 \%(77)$ & $78.4 \%(305)$ & $0.054^{\ulcorner}$ \\
\hline \multicolumn{6}{|c|}{ LDL-cholesterol $<130 \mathrm{mg} / \mathrm{dl}^{2}$} \\
\hline D30 & $69.9 \%(100)$ & $75.9 \%(107)$ & $77.3 \%(68)$ & $73.9 \%(275)$ & $0.372^{\ulcorner}$ \\
\hline M6 & $72.8 \%(110)$ & $71.9 \%(105)$ & $82.6 \%(76)$ & $74.8 \%(291)$ & $0.140^{\circ}$ \\
\hline A1 & $75.0 \%(117)$ & $76.8 \%(109)$ & $75.9 \%(66)$ & $75.8 \%(292)$ & $0.939^{\ulcorner}$ \\
\hline \multicolumn{6}{|c|}{$\mathrm{HDL}$-cholesterol $\geq \mathbf{4 0} \mathrm{mg} / \mathrm{dl}$} \\
\hline D30 & $76.7 \%(112)$ & $76.8 \%(109)$ & $80.5 \%(70)$ & $77.6 \%(291)$ & $0.766^{\ulcorner}$ \\
\hline M6 & $76.7 \%(115)$ & $80.1 \%(117)$ & $78.5 \%(73)$ & $78.4 \%(305)$ & $0.768^{\ulcorner}$ \\
\hline A1 & $79.7 \%(122)$ & $77.1 \%(111)$ & $78.4 \%(69)$ & $78.4 \%(302)$ & $0.857^{\ulcorner}$ \\
\hline \multicolumn{6}{|c|}{ Triglycerides $<150 \mathrm{mg} / \mathrm{dl}$} \\
\hline D30 & $64.8 \%(94)$ & $66.4 \%(93)$ & $63.6 \%(56)$ & $65.1 \%(243)$ & $0.907^{\ulcorner}$ \\
\hline M6 & $57.9 \%(88)$ & $66.0 \%(97)$ & $75.3 \%(70)$ & $65.1 \%(255)$ & $0.021^{\Gamma *}$ \\
\hline A1 & $58.2 \%(89)$ & $65.3 \%(94)$ & $62.5 \%(55)$ & $61.8 \%(238)$ & $0.567^{\ulcorner}$ \\
\hline \multicolumn{6}{|c|}{$\mathrm{HbA} 1 \mathrm{c}<7 \%$} \\
\hline D30 & $89.1 \%(123)$ & $84.9 \%(118)$ & $77.3 \%(68)$ & $84.7 \%(309)$ & $0.054^{\ulcorner}$ \\
\hline M6 & $84.3 \%(118)$ & $87.0 \%(120)$ & $81.3 \%(74)$ & $84.6 \%(312)$ & $0.510^{\ulcorner}$ \\
\hline $\mathrm{A} 1$ & $82.4 \%(126)$ & $82.6 \%(119)$ & $81.8 \%(72)$ & $82.3 \%(317)$ & $0.408^{\ulcorner}$ \\
\hline \multicolumn{6}{|c|}{ Quit smoking if smoker on DO } \\
\hline D30 & $9.5 \%(13)$ & $4.2 \%(6)$ & $1.0 \%(1)$ & $5.3 \%(20)$ & $0.013^{ \pm *}$ \\
\hline M6 & $3.9 \%(5)$ & $4.4 \%(6)$ & $5.0 \%(5)$ & $4.4 \%(16)$ & $0.927^{\ulcorner}$ \\
\hline A1 & $3.4 \%(4)$ & $1.6 \%(2)$ & 0 & $1.8 \%(6)$ & $0.208^{ \pm}$ \\
\hline \multicolumn{6}{|c|}{ Physical activity $\geq 30 \mathrm{~min} 3-5$ times/week } \\
\hline D30 & $52.4 \%(97)$ & $49.4 \%(85)$ & $66.7 \%(70)$ & $54.5 \%(252)$ & $0.015^{r *}$ \\
\hline M6 & $53.9 \%(96)$ & $54.7 \%(94)$ & $62.9 \%(66)$ & $56.3 \%(256)$ & $0.297^{\ulcorner}$ \\
\hline A1 & $47.1 \%(81)$ & $55.4 \%(93)$ & $60.4 \%(61)$ & $53.3 \%(235)$ & $0.082^{r}$ \\
\hline \multicolumn{6}{|c|}{ Sufficient weight reduction ${ }^{3}$ if $\mathrm{BMI} \geq 25$ on $\mathrm{DO}$} \\
\hline D30 & $5.1 \%(7)$ & $3.0 \%(4)$ & $5.7 \%(4)$ & $4.4 \%(15)$ & $0.583^{ \pm}$ \\
\hline M6 & $6.9 \%(9)$ & $3.8 \%(5)$ & $6.8 \%(5)$ & $5.6 \%(19)$ & $0.483^{\ulcorner}$ \\
\hline $\mathrm{A} 1$ & $7.1 \%(9)$ & $6.3 \%(8)$ & $6.8 \%(5)$ & $6.7 \%(22)$ & $0.964^{\ulcorner}$ \\
\hline
\end{tabular}

$B M I$ body mass index

*Significant

${ }^{ \pm}$Fisher's exact test

$\left\ulcorner\mathrm{Chi}^{2}\right.$-Test

${ }^{1}$ If comorbidity diabetes mellitus: $\leq 130 / 80 \mathrm{mmHg}$

${ }^{2}$ If comorbidity coronary heart disease: $<100 \mathrm{mg} / \mathrm{dl}$

${ }^{3}$ If $\mathrm{BMI} 25-27.5$ : Weight reduction to $\mathrm{BMI}<25$, if $\mathrm{BMI}>27.5: 10 \%$ weight reduction; D0: Screening visit; D30: Visit after 30 days; M6: Visit after six months; A1: Visit after one year

$1 \% ; p=0.013)$. The overall rate of patients who had quit smoking went from $5 \%$ at the 30 -day visit to another $4 \%$ at the 6-month visit and another $2 \%$ at the 1 -year visit. When considering all patients, a significant increase in nonsmoking was achieved over time (screening visit: $80 \%$, after one year: $85 \% ; p<0.0001$; see Additional file 1 : Table S2). In the BMT group, patients had significantly more physical activity after one month (BMT: 67\%, CEA: $52 \%$, CAS: $49 \% ; p=0.015$ ), with increasing rates in the CAS group but slightly decreasing rates in the BMT group within one year. At the screening visit, 377 (73.0\%) of all 513 patients had a $\mathrm{BMI} \geq 25$. The number of patients initially overweight at the screening visit with subsequent sufficient weight reduction increased nonsignificantly at a low level, from $4 \%$ after 30 days to $7 \%$ at the one-year visit. For further details, see Table 1.

\section{Control of risk factors}

Relative to the screening visit, a significant reduction in blood pressure (BP, systolic and diastolic) was achieved over time in all patients (median systolic BP D0: $146 \mathrm{~mm}$ Hg, D30: $142 \mathrm{~mm} \mathrm{Hg}(p=0.0005)$, M6: $141 \mathrm{~mm} \mathrm{Hg}$ $(p<0.0001), \mathrm{A} 1: 140 \mathrm{~mm} \mathrm{Hg}(p<0.0001))$. No persistently significant changes in blood glucose or HbA1c levels were observed over time between groups. In terms of lipid parameters, the BMT group had significantly lower cholesterol levels at 1 year (CEA: $174 \mathrm{mg} / \mathrm{dl}$, CAS $167 \mathrm{mg} /$ $\mathrm{dl}$, BMT $164 \mathrm{mg} / \mathrm{dl} ; p=0.0385)$ and significantly lower LDL cholesterol levels at the 6-month visit (CEA: $98 \mathrm{mg} /$ dl, CAS: $95 \mathrm{mg} / \mathrm{dl}, \mathrm{BMT}: 87 \mathrm{mg} / \mathrm{dl} ; p=0.0286$ ) than the intervention groups. Significant reductions in cholesterol and LDL cholesterol were achieved in all patients at each follow-up visit relative to the screening visit (see Table 2 for details). BMI did not differ significantly between groups. Compared with that at the screening visit, the discrete but significant decrease in BMI at the 30-day visit was not maintained in all patients at the 6-month and 1-year visits, and BMI remained at its median of 27. Patients in the BMT group were significantly more likely to be physically active at 30 days (physical activity 3 times or more per week: CEA: 52\%, CAS 49\%, BMT 67\%; $p=0.024)$. The advantage of physical activity in the BMT group persisted at the 6-month and 1-year visits but with no significant difference and decreasing rates. According to the treating physician's assessment, implementation of risk factor modification was significantly better in the BMT group at 6 months (BMT: 76\%, CEA: 68\%, CAS: 54\%; $p<0.001$ ) and at 1 year (BMT: 77\%, CEA: 65\%, CAS: $61 \% ; p=0.027)$. For further details, see Table 2 .

\section{Medication}

Compared with the screening visit, significantly more patients were treated with statin medication after 30 days (D0: 77\%, D30: 88\%; $p<0.0001$ ). This significant difference was maintained at subsequent visits (M6: 90\%, A1: 91\%; $p<0.0001)$. After the screening visit, patients in the 
Table 2 Adjustment of vascular risk factors over time and comparison of study groups; $\mathrm{n}=513$

\begin{tabular}{|c|c|c|c|c|c|c|}
\hline & CEA & CAS & BMT & total & $p^{1}$ & $p^{2}$ \\
\hline Systolic BP DO (median [IQR]) $\square$ & $147(131 ; 160)$ & $145(130 ; 162)$ & $150(133 ; 160)$ & $146(130 ; 160)$ & $0.731>\diamond$ & \\
\hline Diastolic BP D0 (median [IQR]) $\square$ & $80(72 ; 88)$ & $80(74 ; 90)$ & $80(75 ; 89)$ & $80(73 ; 89)$ & $0.5734^{\diamond}$ & \\
\hline Systolic BP D30 (median [IQR]) & $142(131 ; 160)$ & $140(130 ; 154)$ & $141(130 ; 160)$ & $142(130 ; 160)$ & $0.2178 \diamond$ & $0.0005^{\square *}$ \\
\hline Diastolic BP D30 (median [IQR]) & $80(71 ; 85)$ & $80(71 ; 87)$ & $80(70 ; 89)$ & $80(71 ; 86)$ & $0.9233 \diamond$ & $0.0160^{\square *}$ \\
\hline Systolic BP M6 (median [IQR]) & $144.5(130 ; 158)$ & $140(130 ; 159)$ & $140(128 ; 160)$ & $141(130 ; 159)$ & $0.6510 \diamond$ & $<0.0001^{\square *}$ \\
\hline Diastolic BP M6 (median [IQR]) & $80(70 ; 87)$ & $80(74 ; 86)$ & $80(72 ; 86)$ & $80(72 ; 86)$ & $0.4862^{\diamond}$ & $0.0035^{\square *}$ \\
\hline Systolic BP A1 (median [IQR]) & $142.5(130 ; 158)$ & $140(130 ; 156)$ & $140(130 ; 150)$ & $140(130 ; 157)$ & $0.6320 \diamond$ & $<0.0001^{\square *}$ \\
\hline Diastolic BP A1 (median [IQR]) & $80(70.5 ; 85)$ & $80(72 ; 85)$ & $80(70 ; 85)$ & $80(71 ; 85)$ & $0.8059 \diamond$ & $0.0001^{\square *}$ \\
\hline Blood glucose (median [IQR]) Do $\square$ & $103.5(92 ; 121)$ & $104(93 ; 125)$ & $104(92 ; 119)$ & $104(92.5 ; 122)$ & $0.8918 \diamond$ & \\
\hline Blood glucose (median [IQR]) D30 & $110.5(95 ; 131)$ & $105(95 ; 125)$ & $101(92 ; 121)$ & $107(94 ; 125)$ & $0.2776^{\diamond}$ & $0.1054^{\square}$ \\
\hline Blood glucose (median [IQR]) M6 & $108(96 ; 125)$ & $103(94 ; 126)$ & $101(92.5 ; 123)$ & $104(94 ; 125)$ & $0.3761^{\diamond}$ & $0.8950^{\circ}$ \\
\hline Blood glucose (median [IQR]) A1 & $105(93 ; 124)$ & $105(93 ; 124)$ & $105.5(96.5 ; 127)$ & $105(94 ; 124.5)$ & $0.6192^{\diamond}$ & $0.2191^{\square}$ \\
\hline $\mathrm{HbA1c}$ (in \%; median [IQR]) D0 $\square$ & $6(5.7 ; 6.45)$ & $6(5.7 ; 6.6)$ & $5.9(5.55 ; 6.75)$ & $6(5.7 ; 6.5)$ & $0.5940 \diamond$ & \\
\hline HbA1c (in \%; median [IQR]) D30 & $5.9(5.6 ; 6.4)$ & $5.9(5.6 ; 6.4)$ & $5.9(5.5 ; 6.6)$ & $5.9(5.6 ; 6.4)$ & $0.7903 \diamond$ & $0.0025^{\square *}$ \\
\hline $\mathrm{HbA} 1 \mathrm{c}$ (in \%; median [IQR]) M6 & $6.1(5.7 ; 6.6)$ & $6.0(5.7 ; 6.4)$ & $5.9(5.5 ; 6.7)$ & $6.0(5.7 ; 6.5)$ & $0.4636^{\diamond}$ & $0.7925^{\square}$ \\
\hline $\mathrm{HbA} 1 \mathrm{c}$ (in \%; median [IQR]) A1 & $6.0(5.5 ; 6.5)$ & $5.9(5.7 ; 6.5)$ & $6.0(5.5 ; 6.7)$ & $6.0(5.6 ; 6.5)$ & $0.9039 \diamond$ & $0.5419^{\square}$ \\
\hline Cholesterol (median [IQR]) D0 $\square$ & $181(153 ; 209)$ & $173.5(152 ; 200)$ & $169.5(146.5 ; 192.5)$ & $174(151 ; 203)$ & $0.2619 \diamond$ & \\
\hline Cholesterol (median [IQR]) D30 & $175(149 ; 207)$ & $168(150.5 ; 193.5)$ & $159(142 ; 191)$ & $168.5(148 ; 196)$ & $0.0744 \diamond$ & $0.0025^{\square *}$ \\
\hline Cholesterol (median [IQR]) M6 & $176(149 ; 199)$ & $170(150 ; 194)$ & $161(143 ; 175)$ & $168(147 ; 194)$ & $0.0056 \diamond *$ & $0.0091^{\square *}$ \\
\hline Cholesterol (median [IQR]) A1 & $174(150 ; 202)$ & $167(143 ; 198)$ & $164(140 ; 184)$ & $167(146 ; 194)$ & $0.0385^{\diamond *}$ & $0.00170 *$ \\
\hline LDL-Cholesterol (median [IQR]) D0 $\square$ & $105(81 ; 130)$ & $98.5(80 ; 126)$ & $97(80 ; 117)$ & $99(80 ; 123.5)$ & $0.3559 \diamond$ & \\
\hline LDL-Cholesterol (median [IQR]) D30 & $98(78 ; 125)$ & $91(76 ; 114)$ & $88(72 ; 106)$ & $91(76 ; 116)$ & $0.0985^{\diamond}$ & $0.0001^{\square *}$ \\
\hline LDL-Cholesterol (median [IQR]) M6 & $98(75 ; 119)$ & $95(80 ; 115)$ & $87(72 ; 103)$ & $92(75 ; 113)$ & $0.0286^{\diamond *}$ & $0.0006^{\square *}$ \\
\hline LDL-Cholesterol (median [IQR]) A1 & $98(78 ; 118)$ & $93(72 ; 116)$ & $87(69 ; 108)$ & $93(73 ; 116)$ & $0.1164 \diamond$ & $0.0001^{\square *}$ \\
\hline HDL-Cholesterol (median [IQR]) D0 $\square$ & $48(40 ; 62)$ & $49(40 ; 56.5)$ & $49(41.5 ; 57.5)$ & $49(41 ; 58)$ & $0.8799 \diamond$ & \\
\hline HDL-Cholesterol (median [IQR]) D30 & $50(40 ; 60)$ & $48(40 ; 60)$ & $50(41 ; 59)$ & $49(41 ; 60)$ & $0.670 \diamond^{\diamond}$ & $0.8535^{\circ}$ \\
\hline HDL-Cholesterol (median [IQR]) M6 & $49(40 ; 60)$ & $49(41 ; 60)$ & $48(41 ; 56)$ & $49(41 ; 59)$ & $0.8393 \diamond$ & $0.6249^{\circ}$ \\
\hline HDL-Cholesterol (median [IQR]) A1 & $48(41 ; 59.5)$ & $49(40 ; 58.5)$ & $49.5(41.5 ; 60)$ & $49(41 ; 59.5)$ & $0.9204 \diamond$ & $0.0592^{\square}$ \\
\hline Triglycerides (median [IQR]) D0 $\square$ & $131(98 ; 182.5)$ & $128.5(92 ; 184)$ & $120(91 ; 181)$ & $126.5(94 ; 182)$ & $0.6626^{\diamond}$ & \\
\hline Triglycerides (median [IQR]) D30 & $121(92,207)$ & $114(87 ; 174)$ & $121(87 ; 181)$ & $119(88 ; 181)$ & $0.5671^{\diamond}$ & $0.0893^{\square}$ \\
\hline Triglycerides (median [IQR]) M6 & $133.5(94 ; 192)$ & $124(90 ; 164)$ & $107(78 ; 143)$ & $122.5(89 ; 173.5)$ & $0.0123 \diamond *$ & $0.0581^{\square}$ \\
\hline Triglycerides (median [IQR]) A1 & $136(97 ; 192)$ & $112.5(88 ; 182)$ & $132(91 ; 173)$ & $127(91 ; 183)$ & $0.0920 \diamond$ & $0.5763^{\square}$ \\
\hline BMI D0 (median [IQR]) $\square$ & $27(25 ; 30)$ & $27(25 ; 30)$ & $27(24 ; 29)$ & $27(25 ; 30)$ & $0.3567 \diamond$ & \\
\hline BMI D30 (median [IQR]) & $27(25 ; 30)$ & $27(25 ; 30)$ & $26.5(24 ; 29)$ & $27(25 ; 30)$ & $0.1906^{\diamond}$ & $0.0001^{\square *}$ \\
\hline BMI M6 (median [IQR]) & $27(25 ; 30)$ & $27.5(25 ; 30)$ & $27(24 ; 29)$ & $27(25 ; 30)$ & $0.2135^{\diamond}$ & $0.3891^{\circ}$ \\
\hline BMI A1 (median [IQR]) & $27(25 ; 29.5)$ & $27(25 ; 30)$ & $26(24 ; 30)$ & $27(25 ; 30)$ & $0.5458^{\diamond}$ & $0.1201^{\square}$ \\
\hline WHR DO (median [IQR]) & $0.97(0.93 ; 1.02)$ & $0.98(0.94 ; 1.04)$ & $0.99(0.95 ; 1.03)$ & $0.98(0.94 ; 1.03)$ & $0.1514^{\diamond}$ & \\
\hline WHR D30 (median [IQR]) & $0.98(0.93 ; 1.03)$ & $0.98(0.94 ; 1.03)$ & $0.99(0.93 ; 1.03)$ & $0.98(0.93 ; 1.03)$ & $0.7593^{\diamond}$ & $0.8432^{\square}$ \\
\hline WHR M6 (median [IQR]) & $0.98(0.95 ; 1.02)$ & $0.97(0.93 ; 1.02)$ & $1.00(0.94 ; 1.03)$ & $0.98(0.94 ; 1.02)$ & $0.2148^{\diamond}$ & $0.3083^{\circ}$ \\
\hline WHR A1 (median [IQR]) & $0.99(0.95 ; 1.02)$ & $0.98(0.92 ; 1.03)$ & $0.99(0.94 ; 1.03)$ & $0.98(0.94 ; 1.02)$ & $0.6700^{\diamond}$ & $0.7363^{\square}$ \\
\hline \multicolumn{7}{|c|}{ Physical activity > 30 min/week D30 (\% [n]) } \\
\hline None & $24.9 \%(46)$ & $26.2 \%(45)$ & $11.4 \%(12)$ & $22.3 \%(103)$ & $0.024^{\ulcorner *}$ & \\
\hline $1-2$ times & $22.7 \%(42)$ & $24.4 \%(42)$ & $21.9 \%(23)$ & $23.2 \%(107)$ & & \\
\hline 3 times or more & $52.4 \%(97)$ & $49.4 \%(85)$ & $66.7 \%(70)$ & $54.5 \%(252)$ & & \\
\hline \multicolumn{7}{|l|}{ Physical activity > 30 min/week M6 (\% [n]) } \\
\hline None & $20.2 \%(36)$ & $25.6 \%(44)$ & $17.1 \%(18)$ & $21.5 \%(98)$ & $0.267^{\ulcorner}$ & \\
\hline $1-2$ times & $25.8 \%(46)$ & $19.8 \%(34)$ & $20.0 \%(21)$ & $22.2 \%(101)$ & & \\
\hline 3 times or more & $53.9 \%(96)$ & $54.7 \%(94)$ & $62.9 \%(66)$ & $56.3 \%(256)$ & & \\
\hline
\end{tabular}


Table 2 (continued)

\begin{tabular}{|c|c|c|c|c|c|c|}
\hline & CEA & CAS & BMT & total & $p^{1}$ & $p^{2}$ \\
\hline \multicolumn{7}{|l|}{ Physical activity > 30 min/week A1 (\% [n]) } \\
\hline None & $19.8 \%(34)$ & $23.2 \%(39)$ & $16.8 \%(17)$ & $20.4 \%(90)$ & $0.069^{\ulcorner}$ & \\
\hline $1-2$ times & $33.1 \%(57)$ & $21.4 \%(36)$ & $22.8 \%(23)$ & $26.3 \%(116)$ & & \\
\hline 3 times or more & $47.1 \%(81)$ & $55.4 \%(93)$ & $60.4 \%(61)$ & $53.3 \%(235)$ & & \\
\hline Implementation of RFM at D30 (\% [n]) & $74.4 \%(131)$ & $63.9 \%(108)$ & $68.0 \%(70)$ & $69.0 \%(309)$ & 0.104 & \\
\hline Implementation of RFM at M6 (\% [n]) & $67.8 \%(116)$ & $53.7 \%(88)$ & $76.0 \%(79)$ & $64.5 \%(283)$ & $<0.001^{*}$ & \\
\hline Implementation of RFM at A1 (\% [n]) & $64.7 \%(110)$ & $60.8 \%(101)$ & $76.8 \%(76)$ & $66.0 \%(287)$ & $0.027^{\ulcorner *}$ & \\
\hline
\end{tabular}

BP: Blood pressure; BMI: Body mass index; WHR: Waist-hip ratio; all parameters of lipid metabolism and glucose in mg/dl; LDL: Low-density lipoprotein; HDL: Highdensity lipoprotein; RFM: Risk factor modification

* Significant

$\left\ulcorner\mathrm{Chi}^{2}\right.$-Test

$\diamond$ Kruskal-Wallis test

${ }^{-}$Wilcoxon signed-rank test

$\square$ Already published [23]; D30: Visit 30 days after screening visit; M6: Visit 6 months after screening visit; A1: Visit one year after screening visit

${ }^{1}$ testing CEA vs. CAS vs. BMT

2 testing total value D30/M6/A1 vs. screening visit

BMT group had higher but statistically nonsignificant rates of statin medication use (Table 3).

There were no significant differences in antiplatelet medication at any visit relative to the screening visit, except for higher rates of clopidogrel and lower rates of ASA plus dipyridamole in the CAS group because of dual antiplatelet therapy with ASA and clopidogrel before stenting and 1 month afterward. Rates of antiplatelet therapy ranged from 97 to $99 \%$. Patients who did not receive antiplatelet therapy had anticoagulation or short-term ASA interruption due to trauma. Patient anticoagulant use was between 2 and $5 \%$. Most likely, due to changes in the study protocol (after 2013, anticoagulation was no longer an exclusion criterion), there was significantly more use of anticoagulants at low levels during follow-up (D0: 2.3\%, A1: 4.7\%; $p=0.0072$ ). Compared with that at the screening visit, the rate of antihypertensives increased significantly at each visit (D0: $87 \%$, D30: 93\% $(p=0.0015)$, M6: 94\% ( $p=0.0002)$, A1: 96\% $(p<0.0001)$ ).
Beta-blockers were mainly used (54-58\%), followed by ACE inhibitors (49-54\%), diuretics (38-47\%), calcium antagonists (26-33\%) and AT-II antagonists (26-30\%). ACE inhibitors were used significantly more often at 1 month and at 1 year relative to the screening visit. There was also a significant increase in the use of diuretics, calcium antagonists, and beta-blockers. Antidiabetic agents were administered to $25-27 \%$ of patients, without significant rates of increase during follow-up. For details on the prescriptions of the above drug groups, see Additional file 1: Table S3.

\section{Effect of statin medication on lipid levels-intake control} Intake of the prescribed statin medication seems plausible, as cholesterol and LDL cholesterol levels differed significantly $(p<0.0001)$ between patients on and off statin medication at 30 days, 6 months, and at 1 year. LDL cholesterol levels were reduced to $90 \mathrm{mg} / \mathrm{dl}$ or less in

Table 3 Proportion of patients on statin medication in each study group over time; $n=513$

\begin{tabular}{lllllll}
\hline Visit & CEA & CAS & BMT & total & $\boldsymbol{p}^{\mathbf{1}}$ & $\boldsymbol{p}^{\mathbf{2}}$ \\
\hline Screening $\square$ & $80.3 \%(163)$ & $73.6 \%(145)$ & $78.8 \%(89)$ & $77.4 \%(397)$ & $0.257^{\ulcorner}$ & \\
D30 & $87.6 \%(163)$ & $86.7 \%(156)$ & $91.5 \%(97)$ & $88.1 \%(416)$ & $0.456^{\ulcorner}$ & $<0.0001^{\wedge *}$ \\
M6 & $91.7 \%(166)$ & $86.9 \%(152)$ & $93.5 \%(101)$ & $90.3 \%(419)$ & $0.131^{\ulcorner}$ & $<0.0001^{\wedge *}$ \\
A1 & $92.5 \%(160)$ & $87.6 \%(149)$ & $93.2 \%(96)$ & $90.8 \%(405)$ & $0.190^{\ulcorner}$ & $<0.0001^{\wedge *}$ \\
\hline
\end{tabular}

\footnotetext{
${ }^{*}$ Significant

$\left\ulcorner\mathrm{Ch}^{\mathrm{i} 2}\right.$-test

${ }^{\wedge}$ McNemar test

$\square$ Already published [23]; D30: Visit 30 days after screening visit; M6: Visit 6 months after screening visit; A1: Visit o ne year after screening visit

1 testing CEA vs. CAS vs. BMT

${ }^{2}$ testing rate of statin medication on D30/M6/A1 vs. screening visit
} 
statin users. HDL cholesterol levels were higher in the statin user group, but the difference was not significant (Additional file 1: Table S4a-c). No significant association between statin use or cholesterol levels and vascular outcome events was demonstrated.

\section{Discussion}

Within 1 year, 513 patients with asymptomatic carotid stenosis in the SPACE-2 study were analyzed for the implementation of the BMT recommendations. Documentation time points were visits 30 days, 6 months, and 1 year after the screening visit. Although the recommended blood pressure of at least $\leq 130 / 85 \mathrm{~mm} \mathrm{Hg}$ was achieved in only $25-30 \%$ of patients, a significant increase in antihypertensive medication rates from 87 to $96 \%$ with a consecutive significant reduction in blood pressure from a median of $146 / 80 \mathrm{mmHg}$ to $140 / 80 \mathrm{mmHg}$ was demonstrated. One conclusion could be that, even if the recommended treatment goals are not achieved, following the target of given BMT recommendations leads to a significant reduction in blood pressure. Treatment of hypertension is a high priority because it is highly effective in preventing ischemic stroke and is the largest modifiable risk factor for stroke, accounting for one-third of strokes in industrialized countries [5]. Another extremely relevant parameter of stroke prophylaxis is a sufficient adjustment of lipid parameters. Statins have a plaque-stabilizing effect [11] and thus play an important role in stroke prevention [5]. A meta-analysis showed that statin treatment increased over time: $67 \%$ of trials that ended enrollment from 2000 onward reported that at least $25 \%$ of their participants received statins, compared with $4 \%$ of trials that ended enrollment earlier $(p<0.001)$, with a significantly lower incidence of ipsilateral stroke in trials in which $\geq 25 \%$ of patients used statins [17]. Therefore, consistently adjusted statin medication is one of the main components of BMT. In the Asymptomatic Carotid Surgery Trial (ACST), the proportion of patients on lipid-lowering therapy was reported to be less than $10 \%$ in 1993 and increased to more than $80 \%$ by the end of follow-up in 2006-08, with lower rates of stroke and death in patients on lipidlowering therapy [25]. The implementation of recommendations for pharmacological therapy in the routine treatment of ACS was demonstrated in a retrospective single center analysis from 2002 to 2014 with a significant increase in the use of antiplatelet therapy (72\% to 96\%) and statins (13\% to $79 \%$ ) [26]. Due to increased statin use, a risk reduction for cerebral infarction has also been shown in symptomatic carotid stenosis, further emphasizing the importance of medical therapy [27]. However, even in this controlled trial setting, only approximately $75 \%$ of patients achieved sufficient adjustment of the required cholesterol levels. Meanwhile, since the start of the SPACE-2 trial in 2009, the cutoff values for LDL cholesterol were further reduced $(<100 \mathrm{mg} / \mathrm{dl}$ without $\mathrm{CHD},<70 \mathrm{mg} / \mathrm{dl}$ with CHD) [10] or even more strictly reduced to a target of $55 \mathrm{mg} / \mathrm{dl}$ and a reduction in LDL of $>50 \%$ of the initial value for patients in secondary cardiovascular prevention and for patients at very high risk [16]. Because of these stricter targets, recent guidelines recommend the use of more potent CSE inhibitors, the additional administration of ezetimibe, or even PCSK9 inhibitors [28]. Regarding statin medication implementation, we found a significantly increased rate from 77 to $91 \%$ in all patients during the course of the study, with consecutive significant reductions in cholesterol levels (median $174 \mathrm{mg} / \mathrm{dl}$ to $167 \mathrm{mg} / \mathrm{dl}$ ) and LDL cholesterol levels (median $99 \mathrm{mg} / \mathrm{dl}$ to $93 \mathrm{mg} / \mathrm{dl}$ ). Adequate medication compliance could be plausibly demonstrated with a significant reduction in cholesterol and LDL cholesterol levels in patients on statin medication relative to those without. These results are consistent with data from a meta-analysis of 86 randomized controlled trials, which also demonstrated significantly reduced LDL cholesterol, increased rates of statin prescription and improved statin adherence [13]. This study also showed improved LDL cholesterol reduction over time in studies published after 2012. However, this meta-analysis did not identify a single implementation strategy or group of implementation strategies with superior impact to others [13]. In the SPACE- 2 trial, $42 \%$ of all patients smoking at baseline had quit smoking after one year. In the context of this study, this is above the known real-world rate of approximately $20 \%$ within a comparable period [29]. However, SPACE-2 also showed a decrease in motivation over the course of the study, with the rate of patients who quit smoking falling from $5 \%$ after one month to $2 \%$ after one year.

\section{Was the BMT group more motivated?}

The highest rates of statin medication use were found in the BMT group (but with no significant difference from the intervention groups), resulting in significantly lower (LDL)-cholesterol levels in some cases. In addition, patients in the BMT group, in particular, met the prespecified targets for cholesterol levels (with a significant difference from the intervention groups) and LDL/HDL cholesterol levels (without a significant difference). The BMT patients had the best values for lifestyle modification in the form of sufficient physical activity in accordance with the predefined treatment goals. After 6 months and after 1 year, the BMT group showed significantly better implementation of risk factor management as assessed by the treating physician. The intensified optimization of vascular risk factors might possibly have been motivated by the "fear" of progression of stenosis 
or ischemic events, whereas interventionally treated patients felt "safer" after a reduction in stenosis. On the other hand, fewer patients in the BMT group quit smoking, and the rates of adequate weight loss decreased over time. Regarding this inadequate weight reduction and given a recommendation rate for diet of only $54 \%$ and a recommendation rate for sufficient physical activity of $53 \%$ after 1 year, instructions for physical activity and weight reduction should have been emphasized to a greater degree in our study.

The main limitation of the SPACE-2 trial was the premature termination of randomization due to insufficient patient recruitment and a resulting sample size that was too small. Moreover, the statistical concept of this manuscript allows only exploratory, not a confirmatory, analyses. The statistical analyses must therefore be interpreted with caution. Another limitation is the measurement of blood pressure. Since this was performed only once at each visit, a valid interpretation of continuous blood pressure parameters is not possible. The unsatisfactory results in weight reduction may be explained by inadequately implemented nutritional counseling. Taking into account the documentation effort within the scope of the study, measures that were implemented or recommended, such as smoking cessation courses, dietary counseling or fitness training programs, were not recorded. Therefore, a further analysis of the reasons for not achieving the prevention goals was not feasible.

With regard to the analyzed collective, it can be assumed that the patients participating in this study had higher level to reduce vascular risk factors than the collective found in everyday practice. Nevertheless, the improvement in risk modification demonstrated in SPACE-2 through clear goal setting (avoidance of stenosis progression/occurrence of stenosis-related symptoms) and regular visit contacts can also be implemented in a nonstudy setting. Regarding the sustainable effect of the different therapy methods, it could be argued that the interventional methods (CEA/CAS) show a more stable effect after their one-time application than the continuously controlled medical therapy. We showed in this controlled study that BMT can also be maintained at a high level or even improved. In this context, consistent medical therapy must be supplemented with lifestyle modification and continuous motivation for weight reduction and physical activity.

\section{Conclusion}

The SPACE-2 trial demonstrated sustained improvement in noninterventional vascular risk management in patients with ACS treated by general practitioners, internists and neurologists. Target values for cholesterol and HbA1c were best achieved. In this context, a significant increase in statin intake was relevant. Blood pressure control missed the prespecified target, but blood pressure was significantly reduced by intensification of antihypertensive medication. Patients receiving BMT alone without interventional therapy had significantly better adjusted lipid parameters and, in some cases, more physical activity. However, no treatment group achieved sufficient weight reduction. Our data emphasize the importance of individualized treatment and standardized follow-up protocols in all patients at high risk for vascular or cerebral complications. Patients should be informed of detailed treatment goals and of the consequences if they are missed. A comprehensive patient- and family-centered approach might be more efficient [30]. Due to insufficient patient recruitment, the results must be interpreted with caution.

\section{Supplementary Information}

The online version contains supplementary material available at https://doi. org/10.1186/s42466-021-00153-w.

Additional file 1. Table S1: Prespecified treatment goals for vascular risk factors in SPACE-2. Table S2: Nonsmoking rates and avoidance of obesity over time. Table S3: Medication from screening visit to one year. Table S4 $\mathrm{a} / \mathrm{b} / \mathrm{c}$ : Lipid level as a function of statin intake after 30 days, 6 months and one year.

Members of SPACE-2 steering committee

D. Böckler, M. Böhm, H. Brückmann, E. S. Debus, J. Fiehler, K. Mathias, W. Lang, E. B. Ringelstein, J. Schmidli, R. Stingele, R. Zahn.

\section{Principal investigators and further study personnel}

T. Zeller, W. D. Niesen, K. Barlinn, A. Binder, J. Glahn, F. Beyersdorf, M. Grügerny, R.-R. Macharzina, G. Lechner, C. Menz, S. Schonhardt, M. Weinbeck, O. Greb, D. Otto, T. Winker, H. Berger, H. Poppert, V. Pütz, K. Haase, U. Bodechtel, N. Weiss, H. Bergert, J. Meyne, J. Groß, A. Botsch, M. Kruse, B. Gerdes, W. D. Reinbold, H. Wuttig, A. Maier-Hasselmann, M. Segerer, H.-H. Fuchs, S. Gass, H. Schultz, C. Groden, M. Niedergethman, M. Griebe, M. Rosenkranz, C. Beck, G. Thomalla, H. Zeumer, M. Jauß, W. Kneist, M. Kneist, T. Staudacher, A. Bernhard, D. Jost, N. Prey, J. Knippschild, O. Kastrup, M. Köhrmann, B. Frank, V. Bongers, J. Hoffmann, H.-W. Kniemeyer, M. Knauth, K. Wasser, T. Stojanovic, H. Emmert, J. Tacke, B. Schwalbe, E.-M. Nam, U. van Lengerich, S. Lowens, K. Gröschel, T. Uphaus, S. Gröschel, S. Boor, B. Dorweiler, E. Schmid, H. Henkes, T. Hupp, O. Singer, G. Hamann, M. Wagner-Heck, S. Kerth-Krick, M. Kilic, P. Huppert, K. Niederkorn, J. Fruhwirth, G. Klein, U. Pulkowski, K. Jöster, J.-H. Wacks, E. Kloppmann, B. Vatankhah, S. Hopf-Jensen, H. Stolze, S. Müller-Hülsbeck, K. P. Walluscheck, H. M. Schmitt, A. Grüger, J. Seemann, B. Tilahun, M. Dichgans, F. Wollenweber, A. Dörr, A. Zollver, G. Gäbel, G. Hedtmann, R. Kollmar, D. Claus, C. Petermann, S. Kirsch, B. Bosnjak, J. Heiß, H. Mühling, S. Wunderlich, P. N. Sabisch, G. Gahn, M. Storck, S. Arnold, U. Fischer, J. Gralla, M. von Mering, R. Dißmann, D. Kirsch, C. Schmidauer, P. Waldenberger, M. Furtner, H. Kazarians, P. Breuer, C. Arning, J. Rieper, G. Schmidt, M. Arnold, G. Schroth, J. Weise, J. Zanow, T. Mayer, R. Töpper, W. Gross-Fengels, H. Daum, R. Dittrich, M. Ritter, B. Kasprzak, G. Torsello, C. Pohlmann, R. Brüning, P. Breuer, H. Amiri, I. Ludwig, E. Blessing, M. Möhlenbruch, A. Crispin, M. Hofman, T. Müller.

\section{Authors' contributions}

Hacke W: Coordinating investigator, conception and design of the study, final approval of the version to be published, member of the Executive Committee (EC), and member of the Steering Committee (SC). Ringleb PA: Study coordinator, conception and design of the study, cleaning and interpretation of the data, manuscript writing, final approval of the version to be published, 
member of the EC, and member of the SC. Eckstein $\mathrm{H}-\mathrm{H}$ : Principal coinvestigator, conception and design of the study, interpretation of the data, manuscript writing, final approval of the version to be published, member of the EC, and member of the SC. Mansmann U: Design of the data collection tools, monitoring of the data collection, conception and design of the study, cleaning and statistical analysis of the data, interpretation of the data, manuscript writing, final approval of the version to be published, member of the $\mathrm{EC}$, and member of the SC. Jansen O: Principal coinvestigator, conception and design of the study, interpretation of the data, manuscript writing, final approval of the version to be published, member of the EC and member of the SC. Fraedrich G: Conception and design of the study, interpretation of the data, manuscript writing, final approval of the version to be published, member of the EC, and member of the SC. Mudra H: Conception and design of the study, interpretation of the data, manuscript writing, final approval of the version to be published, member of the EC, and member of the SC. Reiff T: Study coordinator, monitoring data collection, conception and design of the study, cleaning and statistical analysis of the data, interpretation of the data, manuscript writing, submitting manuscript, and final approval of the version to be published. All authors read and approved the final manuscript.

\section{Funding}

BMBF/DFG (HA 1394/5-1).

\section{Declarations}

\section{Ethics approval and consent to participate}

The study was approved by the ethics committee of University Heidelberg (S-311/2008) and the local ethic committees of the participating centers.

\section{Consent for publication}

Informed consent was obtained from all individual participants included in the study.

\section{Competing interest}

The authors declare that they have no competing interests.

\section{Author details}

'Department of Neurology, University Hospital of Heidelberg, Im Neuenheimer Feld 400, 69120 Heidelberg, Germany. ${ }^{2}$ Department for Vascular and Endovascular Surgery, Klinikum Rechts Der Isar, Technical University of Munich, Munich, Germany. ${ }^{3}$ Institute of Medical Informatics, Biometry and Epidemiology, Ludwig-Maximilians-Universität München, Munich, Germany. ${ }^{4}$ Department of Radiology and Neuroradiology, UKSH Campus Kiel, Kiel, Germany. ${ }^{5}$ Department of Vascular Surgery, University Hospital of Innsbruck, Innsbruck, Austria. ${ }^{6}$ Department of Cardiology, Klinikum Neuperlach, München KlinikMunich, Germany.

Received: 14 July 2021 Accepted: 24 August 2021

Published online: 19 October 2021

\section{References}

1. Greco, G., Egorova, N. N., Moskowitz, A. J., Gelijns, A. C., Kent, K. C., Manganaro, A. J., et al. (2013). A model for predicting the risk of carotid artery disease. Annals of Surgery, 257(6), 1168-1173. https://doi.org/10.1097/SLA. Ob013e31827b9761

2. Nicolaides, A. N., Kakkos, S. K., Kyriacou, E., Griffin, M., Sabetai, M., Thomas, D. J., et al. (2010). Asymptomatic internal carotid artery stenosis and cerebrovascular risk stratification. Journal of Vascular Surgery, 52(6), 1486-1496. https://doi.org/10.1016/j.jvs.2010.07.021

3. Poorthuis, M. H. F., Halliday, A., Massa, M. S., Sherliker, P., Clack, R., Morris, D. R., et al. (2020). Validation of risk prediction models to detect asymptomatic carotid stenosis. Journal of the American Heart Association, 9(8), e014766. https://doi.org/10.1161/JAHA.119.014766

4. O’Donnell, M. J., Chin, S. L., Rangarajan, S., Xavier, D., Liu, L., Zhang, H., et al. (2016). Global and regional effects of potentially modifiable risk factors associated with acute stroke in 32 countries (INTERSTROKE): A case-control study. Lancet, 388(10046), 761-775. https://doi.org/10.1016/ s0140-6736(16)30506-2
5. Diener, H. C., \& Hankey, G. J. (2020). Primary and secondary prevention of ischemic stroke and cerebral hemorrhage: JACC focus seminar. Journal of the American College of Cardiology, 75(15), 1804-1818. https://doi.org/10. 1016/j.jacc.2019.12.072

6. Paraskevas, K. I., Mikhailidis, D. P., Veith, F. J., \& Spence, J. D. (2016). Definition of best medical treatment in asymptomatic and symptomatic carotid artery stenosis. Angiology, 67(5), 411-419. https://doi.org/10.1177/ 0003319715624526

7. Spence, J. D. (2018). Advances in stroke prevention. Journal of Translation Internal Medicine, 6(3), 105-114. https://doi.org/10.2478/jtim-2018-0024

8. Murphy, S. J. X., Naylor, A. R., Ricco, J. B., Sillesen, H., Kakkos, S., Halliday, A., et al. (2019). Optimal antiplatelet therapy in moderate to severe asymptomatic and symptomatic carotid stenosis: A comprehensive review of the literature. European Journal of Vascular and Endovascular Surgery, 57(2), 199-211. https://doi.org/10.1016/j.ejvs.2018.09.018

9. Guirguis-Blake, J. M., Evans, C. V., Senger, C. A., O'Connor, E. A., \& Whitlock, E. P. (2016). Aspirin for the primary prevention of cardiovascular events: A systematic evidence review for the US preventive services task force. Annals of Internal Medicine, 164(12), 804-813. https://doi.org/10.7326/ M15-2113

10. Eckstein, H.-H., Kühnl, A., Berkefeld, J., Dörfler, A., Kopp, I., Langhoff, R., et al. (2020). S3-Leitlinie zur Diagnostik, Therapie und Nachsorge der extracraniellen Carotisstenose. AWMF-Registernummer: 004-028. 2020. https://www.awmf.org/leitlinien/detail/II/004-028.html.

11. Spence, J. D., Coates, V., Li, H., Tamayo, A., Munoz, C., Hackam, D. G., et al. (2010). Effects of intensive medical therapy on microemboli and cardiovascular risk in asymptomatic carotid stenosis. Archives of Neurology, 67(2), 180-186. https://doi.org/10.1001/archneurol.2009.289

12. Martin-Ruiz, E., Olry-de-Labry-Lima, A., Ocana-Riola, R., \& Epstein, D. (2018). Systematic review of the effect of adherence to statin treatment on critical cardiovascular events and mortality in primary prevention. Journal of Cardiovascular Pharmacology and Therapeutics, 23(3), 200-215. https://doi.org/10.1177/1074248417745357

13. Jones, L. K., Tilberry, S., Gregor, C., Yaeger, L. H., Hu, Y., Sturm, A. C., et al. (2021). Implementation strategies to improve statin utilization in individuals with hypercholesterolemia: a systematic review and metaanalysis. Implementation Science, 16(1), 40. https://doi.org/10.1186/ s13012-021-01108-0

14. Lansberg, P., Lee, A., Lee, Z. V., Subramaniam, K., \& Setia, S. (2018). Nonadherence to statins: Individualized intervention strategies outside the pill box. Vascular Health and Risk Management, 14, 91-102. https://doi.org/10. 2147NHRM.S158641

15. Paraskevas, K. I., Geroulakos, G., Veith, F. J., \& Mikhailidis, D. P. (2020). Multifocal arterial disease: Clinical implications and management. Current Opinion in Cardiology, 35(4), 412-416. https://doi.org/10.1097/HCO.00000 00000000746

16. Messas, E., Goudot, G., Halliday, A., Sitruk, J., Mirault, T., Khider, L., et al. (2020). Management of carotid stenosis for primary and secondary prevention of stroke: State-of-the-art 2020: a critical review. European Heart Journal Supplements 2020;22 Suppl M:M35-M42; doi:https://doi.org/10. 1093/eurheartj/suaa162.

17. Hadar, N., Raman, G., Moorthy, D., O'Donnell, T. F., Thaler, D. E., Feldmann, E., et al. (2014). Asymptomatic carotid artery stenosis treated with medical therapy alone: Temporal trends and implications for risk assessment and the design of future studies. Cerebrovascular Diseases, 38(3), 163-173. https://doi.org/10.1159/000365206

18. Randomised trial of endarterectomy for recently symptomatic carotid stenosis: final results of the MRC European Carotid Surgery Trial (ECST). Lancet. 1998;351(9113), 1379-1387. doi: S0140673697092921.

19. Barnett, H. J. M., Taylor, D. W., Haynes, R. B., Sackett, D. L., Peerless, S. J., Ferguson, G. G., et al. (1991). Beneficial effect of carotid endarterectomy in symptomatic patients with high-grade carotid stenosis. New England Journal of Medicine, 325(7), 445-453. https://doi.org/10.1056/NEJM199108 153250701

20. Reiff, T., Stingele, R., Eckstein, H. H., Fraedrich, G., Jansen, O., Mudra, H., et al. (2009). Stent-protected angioplasty in asymptomatic carotid artery stenosis vs. endarterectomy: SPACE2: A three-arm randomised-controlled clinical trial. International Journal of Stroke, 4(4):294-299. doi: https://doi. org/10.1111/j.1747-4949.2009.00290.x 
21. Reiff, T., Amiri, H., Eckstein, H. H., Fraedrich, G., Jansen, O., Mudra, H., et al. (2014). Modification of SPACE-2 study design. International Journal of Stroke, 9(3), E12-E13. https://doi.org/10.1111/ijs.12253

22. Eckstein, H. H., Reiff, T., Ringleb, P., Jansen, O., Mansmann, U., \& Hacke, W. (2016). SPACE-2: A missed opportunity to compare carotid endarterectomy, carotid stenting, and best medical treatment in patients with asymptomatic carotid stenoses. European Journal of Vascular and Endovascular Surgery, 51(6), 761-765. https://doi.org/10.1016/j.ejvs.2016 02.005

23. Reiff, T., Eckstein, H. H., Mansmann, U., Jansen, O., Fraedrich, G., Mudra, $H_{\text {., }}$ et al. (2020). Angioplasty in asymptomatic carotid artery stenosis vs. endarterectomy compared to best medical treatment: One-year interim results of SPACE-2. International Journal of Stroke, 15(6), 638-49. doi: https://doi.org/10.1177/1747493019833017.

24. Sacco, R. L., Adams, R., Albers, G., Alberts, M. J., Benavente, O., Furie, K., et al. (2006). Guidelines for prevention of stroke in patients with ischemic stroke or transient ischemic attack: A statement for healthcare professionals from the American Heart Association/American Stroke Association Council on Stroke: Co-sponsored by the Council on Cardiovascular Radiology and Intervention: The American Academy of Neurology affirms the value of this guideline. Stroke, 37(2), 577-617. https://doi.org/10.1161/ 01.STR.0000199147.30016.74

25. Halliday, A., Harrison, M., Hayter, E., Kong, X., Mansfield, A., Marro, J., et al. (2010). 10-year stroke prevention after successful carotid endarterectomy for asymptomatic stenosis (ACST-1): A multicentre randomised trial. Lancet, 376(9746), 1074-1084. https://doi.org/10.1016/S0140-6736(10) 61197-X

26. Bissacco, D., Carmo, M., Barbetta, I., Dallatana, R., \& Settembrini, P. G. (2018). Medical therapy before carotid endarterectomy: Changes over a 13-year period and comparison between asymptomatic and symptomatic patients. Angiology, 69(2), 113-119. https://doi.org/10.1177/ 0003319717706626

27. Fisch, U., von Felten, S., Wiencierz, A., Jansen, O., Howard, G., Hendrikse, J., et al. (2021). Risk of stroke before revascularisation in patients with symptomatic carotid stenosis: A pooled analysis of randomised controlled trials. European Journal of Vascular and Endovascular Surgery. https://doi. org/10.1016/j.ejvs.2021.02.024

28. Authors/Task Force M, Guidelines ESCCFP, Societies ESCNC. 2019 ESC/EAS guidelines for the management of dyslipidaemias: Lipid modification to reduce cardiovascular risk. Atherosclerosis 2019;290:140-205; doi:https:// doi.org/10.1016/j.atherosclerosis.2019.08.014.

29. Watkins, S. L., Thrul, J., Max, W., \& Ling, P. M. (2020). Real-world effectiveness of smoking cessation strategies for young and older adults: Findings from a Nationally Representative Cohort. Nicotine \& Tobacco Research, 22(9), 1560-1568. https://doi.org/10.1093/ntr/ntz223

30. Piepoli, M. F., Hoes, A. W., Agewall, S., Albus, C., Brotons, C., Catapano, A. L., et al. (2016). 2016 European Guidelines on cardiovascular disease prevention in clinical practice: The Sixth Joint Task Force of the European Society of Cardiology and Other Societies on Cardiovascular Disease Prevention in Clinical Practice (constituted by representatives of 10 societies and by invited experts)Developed with the special contribution of the European Association for Cardiovascular Prevention \& Rehabilitation (EACPR). European Heart Journal, 37(29), 2315-2381. https://doi.org/10.1093/eurhe artj/ehw106

\section{Publisher's Note}

Springer Nature remains neutral with regard to jurisdictional claims in published maps and institutional affiliations.
Ready to submit your research? Choose BMC and benefit from:

- fast, convenient online submission

- thorough peer review by experienced researchers in your field

- rapid publication on acceptance

- support for research data, including large and complex data types

- gold Open Access which fosters wider collaboration and increased citations

- maximum visibility for your research: over $100 \mathrm{M}$ website views per year

At BMC, research is always in progress.

Learn more biomedcentral.com/submissions 